\title{
Optimization of the Extent of Surgical Treatment in Patients with Stage $I$ in Cervical Cancer
}

\author{
A. L. Chernyshova ${ }^{1}$, L. A. Kolomiets ${ }^{1,3}$, I. G. Sinilkin ${ }^{1,2}$, \\ V. I. Chernov ${ }^{1,2}$, and A. Yu. Lyapunov ${ }^{1, a)}$ \\ ${ }^{1}$ Tomsk Cancer Research Institute, Kooperativny Street 5, Tomsk, 634050 Russia \\ ${ }^{2}$ Tomsk Polytechnic University, Lenin Avenue 30, Tomsk, 634050 Russia \\ ${ }^{3}$ Siberian State Medical University, Moskovsky Trakt 2, Tomsk, 634050 Russia

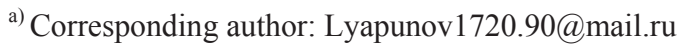

\begin{abstract}
The study included 26 patients with FIGO stage Ia1-Ib1 cervical cancer who underwent fertility-sparing surgery (transabdominaltrachelectomy). To visualize sentinel lymph nodes, lymphoscintigraphy with injection of $99 \mathrm{mTc}$ labelled nanocolloid was performed the day before surgery. Intraoperative identification of sentinel lymph nodes using hand-held gamma probe was carried out to determine the radioactive counts over the draining lymph node basin. The sentinel lymph node detection in cervical cancer patients contributes to the accurate clinical assessment of the pelvic lymph node status, precise staging of the disease and tailoring of surgical treatment to individual patient.
\end{abstract}

\section{INTRODUCTION}

Cervical cancer is the 7 th most common cancer worldwide, accounting for $9.8 \%$ of all female cancers. About 15000 new cervical cancers are estimated to be diagnosed in Russia every year. Cervical cancer is the most common among women aged between 40 and 60 with the incidence rate showing an increasing tendency in women diagnosed with cervical cancer during their childbearing years. The tendency towards increased incidence is also observed among women of the Siberian region of Russia [1,2]. Because of this trend, there has been an increased emphasis on the development of innovative methods and new technologies in the treatment of cervical cancer, as evidenced by numerous publications presented in world literature [3, 4].

For many malignancies affecting the internal organs and for cervical cancer, in particular, preoperative staging assessment is not definitive. At present, the histopathological examination of the surgical specimen is the only reliable method, which determines the status of regional lymph nodes and tumor staging.

Recently, abdominal radical trachelectomy has become more widely used in gynecologic oncology centers for treating young women with FIGO stage IA2-IB2 invasive cervical cancer and tumor size of $\leq 6 \mathrm{~cm}[4-6]$. In some cases, abdominal radical trachelectomy can be used for stage 1B2-IIA tumors of less than $4 \mathrm{~cm}$ in size and having no evidence of metastasis [7].

Radical abdominal trachelectomy (RAT) is a surgical procedure that removes the cervix, upper one-third of the vagina and lymph nodes in the pelvis, while preserving the ovaries, fallopian tubes and the uterine corpus that enables subsequent pregnancy and childbirth. Pelvic lymphodenectomy is performed fist and the removed lymph nodes are sent for routine pathological examination. If lymph nodes are positive, radical hysterectomy with ovarian transposition is performed. If cancer cells are not found in lymph nodes, the cervix with paracervical, parametrial tissues and upper one-third of the vagina are removed. A negative proximal resection margin should be histologically confirmed $[4,8]$.

The sentinel lymph node (SLN) is the first node to which metastatic disease will spread from a primary tumor. If the SLN is free of metastatic disease, all other lymph nodes will also be free of them. Complete pelvic and 
paraaorticlymphadenectomy may cause severe surgical complications, which can lengthen the duration of hospital stay and considerably affect the quality of life of the patients [9-11].

Identification of SLNs may help some patients avoid more extensive lymph node surgery, thus minimizing postoperative morbidity and significantly improving life quality of the patients $[3,12,13]$.

The sentinel lymph node detection contributes to the accurate clinical assessment of the lymph node status, precise staging of the disease and tailoring of surgical treatment to individual patient $[11,14,15]$.

The aim of this study was to evaluate the role of SLN detection using preoperative lymphoscintigraphy and intraoperative gamma probe and to determine the extent of surgery in organ-preserving treatment for invasive cervical cancer.

\section{MATERIALS AND METHODS}

Between 2010 and 2014, 26 women of reproductive age with stage I cervical cancer underwent radical transabdominaltrachelectomy. Out of the 26 patients with stage I cervical cancer, there were 7 (27\%) with stage Ia1, $8(31 \%)$ with stage Ia2 and $11(42 \%)$ with stage Ib1. Non-keratinizing squamous cell carcinoma was histologically proven in all patients. The mean age of the patients was $28.7 \pm 4.5$ years.

All patients were submitted to preoperative lymphoscintigraphy with 99mTc-labelled nanocolloid and intraoperative SLN detection using hand-held gamma probe Gamma Finder II (Germany).

The day before surgery, four injections of $99 \mathrm{mTc}$-labelled nanocolloid at a dose of $20 \mathrm{MBq}$ per quadrant in a volume of $0.25 \mathrm{ml}$ per site were made in each quadrant around the cervical tumor. Lymphoscintigraphy consisted in obtaining an early image ( $20 \mathrm{~min}$ after injection) and a late image ( $3 \mathrm{~h}$ after injection), in 32 views (low-energy, high-resolution, parallel-hole collimator, static image $(64 \times 64)$. The exposure time per projection was $20 \mathrm{~s}$. The data obtained were processed using E.Soft (Siemens) software providing three-dimensional images.

For intraoperative detection of sentinel lymph nodes, the hand-held collimated Gamma Finder II probe was used, which allowed the surgeon to precisely locate gamma radiation source and obtain accurate information about the distribution of the radionuclide in the tissues and organs of the patient. Intraoperative identification of sentinel lymph nodes was performed after opening the retroperitoneal space. The lymph node with radioactivity of at least three times more than the background counts was defined as a sentinel lymph node [16]. The sentinel lymph node was marked and separately sent for express cytological examination. The lymph node was sectioned transversely into $2 \mathrm{~mm}$ slices. Depending on the size of the node, 6-10 imprints were made from each slice by gently touching the cut surface of the node onto a glass slide. Cytological specimens were stained for $15 \mathrm{~s}$ using the kit for rapid staining of blood smears (Leucodiff 200) and examined with a Zeiss Axio Scope.

Then the patients underwent iliac-pelvic lymph node dissection. After lymph node dissection, the removed lymph nodes were re-examined with gamma probe to detect SLNs missed during intraoperative examination (Fig. 1b). The true number of SLNs was calculated as the sum of SLNs identified intraoperatively and in the surgical specimen.

\section{RESULTS AND DISCUSSION}

Single-photon emission computed tomography (SPECT) of the pelvis and abdomen allowed detection of SLNs in $21(80.8 \%)$ of patients. A total of 45 nodes were detected by gamma probe during surgery in all 26 patients. Two nodes that were missed intraoperatively were subsequently detected in a surgical specimen (Fig. 1).

Sentinel lymph nodes were found most frequently in the common iliac region $(37.7 \%)$ and in descending order of frequency, in the external and internal iliac regions $(28.8 \%$ and $17.4 \%$, respectively), in the obturator region $(9.6 \%)$ and in the parametrial region $(6.5 \%)$. The excised SLNs were intraoperatively evaluated by imprint cytology with subsequent routine histological examination of all excised lymph nodes (Table 1). Intraoperative cytology revealed metastatic involvement of SLNs only 2 patients. The extent of surgical treatment for these patients was increased to radical hysterectomy with ovarian transposition.

The comparison between the two techniques of SLN detection revealed that the intraoperative gamma probe detection technique was more effective than SPECT, showing a sensitivity rate of $93.3 \%$ compared to $72 \%$. According to a number of studies conducted in leading hospitals around the world, the combined use of the radiopharmaceutical tracer and blue dye (isosulfan blue) is the optimal method for SLN detection. Detection by 99mTc-nanocolloid resulted in a higher yield of SLNs than detection with blue dye with sensitivity rates of $88.5 \%$ and $83.9 \%$, respectively. The combined technique appears to be the most sensitive diagnostic technique, when the likelihood of SLN detection increases to $91.4 \%[17,18]$. 


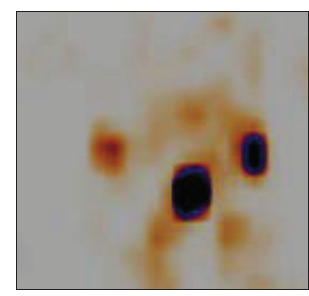

(a)

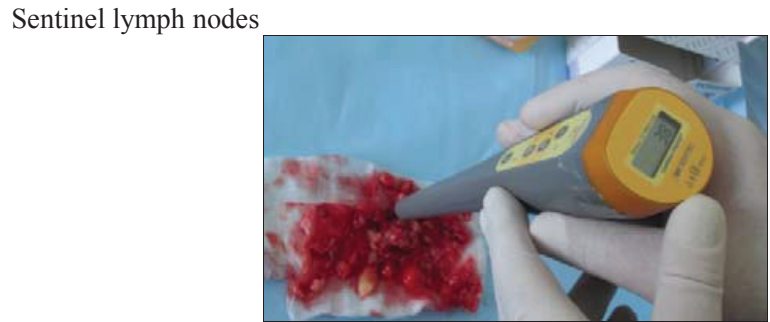

(b)

FIGURE 1. (a) Sentinel lymph node visualization (arrow) on SPECT image, (b) gamma probe identification of sentinel lymph nodes in the biopsy specimen

A number of studies devoted to the examination of SLN in gynecological cancer are currently conducted [16, 19, 20]. In 2008, the results of European multi-center study conducted by the National Cancer Institute (Paris) were published. They were dedicated to the study of the prognostic significance and anatomical distribution of SLNs in cervical cancer. Sentinel lymph nodes were identified using a combination of 99mTc-nanocolloid + isosulfan blue followed by complete pelvic and praraaortic lymphadenectomy. Sentinel lymph nodes were detected in $96.1 \%$ of patients, $5.5 \%$ of whom were found to have metastases in SLNs, and no positive non-SLNs were present in negative SLNs patients. The most common site for SLN detection wasthe external iliac (83.5\%). Only $8.5 \%$ SLNs were located in the common iliac, $5.1 \%$ in the presacral and retrosacral areas and $2.7 \%$ in the parametrial region [20].

Plentl et al. [21] found the SLNs in the parametrial region. SLNs in this location cannot be identified easily by gamma probe during surgery because of the proximity of the cervical tumor and the peritumoral area of increased radioactivity. The most common site for SLN detection was the obturator fossa $(50.4 \%)$, followed by the external iliac artery $(31.4 \%)$, the internal iliac artery $(15.2 \%)$, the common iliac artery $(6 \%)$, and the cardinal ligament (2 nodes). Unilateral SLNs were detected in 52.9\%. Possible explanations can be argued that the lymphatic drainage of SLN could occur in one side of the pelvis.

Similar results were obtained by H. Hertel et al. (Sunnybrook Health Sciences Center, Toronto). SLNs were detected in $85 \%$ of cases, metastases in SLNs were found in $10 \%$ of patients, and no cases with positive non-SLNs with the presence of negative SLNs were revealed. The authors reported sensitivity of $92 \%$. To date, in published studies, sensitivity ranges from $71 \%$ to $100 \%$, diagnostic accuracy from $75 \%$ to $100 \%$ with specificity of $100 \%$ [12].

\section{CONCLUSION}

Sentinel lymph node mapping in cervical cancer complements current trends in minimally invasive surgery, however, this technique has not yet found wide acceptance in clinical practice as a component of a comprehensive examination and treatment of the patients with cervical cancer. Further research is needed to optimize the staging of regional dissemination of tumors and, therefore, the choice of adequate treatment [2, 21, 22]. Sentinel node biopsy may be a reasonable alternative to avoid extensive pelvic lymph nodes dissection in early stage cervical cancer patients with negative SLNs. In contrast, the detection of SLN metastasis during surgery calls for pelvic lymphadenectomy to be extended to the para-aortic area. However, larger randomized studies are required before recommending routine use of the SLN approach to the determination of the extent of surgical treatment.

TABLE 1. Location of SLNs detected by ${ }^{99 \mathrm{~m}}$ Tc-labelled nanocolloid

\begin{tabular}{lccc}
\hline \multicolumn{1}{c}{ Location of lymph nodes } & $\begin{array}{c}\text { On the right side } \\
\boldsymbol{n = 6 ( 1 3 \% )}\end{array}$ & $\begin{array}{c}\text { On the left side } \\
\boldsymbol{n = 4}(\mathbf{1 0} \%)\end{array}$ & $\begin{array}{c}\text { Bilaterally } \\
\boldsymbol{n}=\mathbf{3 5}(\mathbf{7 7 \%})\end{array}$ \\
\hline Common iliac artery & 3 & 2 & 12 \\
\hline External iliac artery & 1 & 2 & 10 \\
\hline Internal iliac artery & 0 & 0 & 8 \\
\hline Obturator fossa & 1 & 0 & 3 \\
\hline Parametrial tissue & 0 & 0 & 3 \\
\hline
\end{tabular}




\section{ACKNOWLEDGMENTS}

The study reported in this article was conducted according to accepted ethical guidelines involving research in humans and/or animals and was approved by an appropriate institution or national research organization. The study is compliant with the ethical standards as currently outlined in the Declaration of Helsinki. All individual participants discussed in this study, or for whom any identifying information or image has been presented, have freely given their informed written consent for such information and/or image to be included in the published article.

\section{REFERENCES}

1. E. L. Choynzonov, L. F. Pisareva, N. V. Cherdyntseva, A. P. Boyarkina, I. N. Odintsova, and N. A. Martynova, Cancer incidence in Siberia and Russian Far East. Cancer care service and ways of its improvement, Bullet. Siberian Branch of the Russian Academy of Medical Sciences 2, 41-47 (2004).

2. E. L. Choynzonov, L. F. Pisareva, and L. D. Zhuikova, Cancer incidence in the Tomsk region during the period 2004-2009. Assessment of diagnostic quality, Siberian J. Oncology 3, 29-34 (2011).

3. V. F. Semiglazov, New Trend Sinorgan-Sparing Surgery for Cancer (St-Petersburg, 2009), pp. 12-24.

4. A. L. Chernyshova, L. A. Kolomiets, and S. E. Krasilnikov, Organ-preserving treatment for invasive cervical cancer, Siberian J. Oncology 51(2), 72-78 (2011).

5. N. R. Abu-Rustum, N. Neubauer, Y. Sonoda, et al., Surgical and pathologic outcomes of fertility-sparing radical abdominal trachelectomy for FIGO stage IB1 cervical cancer, Gynecol. Oncol. 111(2), 261-264 (2008).

6. H. Hertel, C. Köhler, and D. Grund, Association of Gynecologic Oncologists (AGO): Radical vaginal trachelectomy (RVT) combined with laparoscopic pelvic lymphadenectomy: prospective multicenter study of 100 patients with early cervical cancer, Gynecol. Oncol. 103(2), 506-511 (2006).

7. J. P. Diaz, Y. Sonoda, and M. M. Leitao, Oncologic outcome of fertility-sparing radical trachelectomy versus radical hysterectomy for stage IB1 cervical carcinoma, Gynecol. Oncol. 111(2), 255-260 (2008).

8. T. Yamashita, H. Katayama, and Y. Kato, Management of pelvic lymph nodes by sentinel node navigation surgery in the treatment of invasive cervical cancer, Int. J. Gynecol. Cancer 19(6), 1113-1118 (2009).

9. C. Altgassen and H. Hertel, Multicenter validacion study of the sentinentel lymph node concept in cervical cancer: AGO Study Group, J. Clin. Oncol. 26, 2943-2951 (2008).

10. C. Levenback, Update on sentinel lymph node biopsy in gynecologic cancers, Gynecol. Oncol. 111(Suppl. 2), S42S43 (2008)

11. I. Sinilkin, V. Chernov, R. Zelchan, A. A. Titskaya, and V. Skuridin, Clinical investigation of nanocolloid 99m$\mathrm{Al}_{2} \mathrm{O}_{3}$ for sentinel lymph nodes visualization, in Congress of the European Association of Nuclear Medicine, Gothenburg, Sweden 18-22 Oct. 2014, Eur. J. Nucl. Med. Mol. Imaging 41(Suppl 2), S518 (2014).

12. A. L. Chernyshova, A. Yu. Lyapunov, L. A. Kolomiets, V. I. Chernov, and I. G. Sinilkin, Sentinellymphnode detection during surgery for cervical cancer, Siberian J. Oncology 51(3), 28-33 (2012).

13. S. J. Seong, H. Park, and K. M. Yang, Detection of sentinel lymph nodes in patients with early stage cervical cancer, J. Korean Med. Sci. 22(1), 105-109 (2007).

14. L. Darlin, J. Persson, and T. Bossmar, The sentinel node concept in early cervical cancer performs well in tumors smaller than $2 \mathrm{~cm}$, Gynecol. Oncol. 117(2), 266-269 (2010).

15. L. R. Eiriksson and A. Covens, Sentinel lymph node mapping in cervical cancer: the future? Int. J. Obstetrics Gynaecology 119(2), 129-133 (2012).

16. S. Ogawa, H. Kobayashi, and S. Amada, Sentinel node detection with $(99 \mathrm{~m})$ Tcphytate alone is satisfactory for cervical cancer patients undergoing radical hysterectomy and pelvic lymphadenectomy, Int. J. Clin. Oncol. 15(1), 52-58 (2010).

17. P. P. Kara, A. Ayhan, and B. Caner, Sentinel lymph node detection in early stage cervical cancer: a prospective study comparing preoperative lymphoscintigraphy, intraoperative gamma probe, and blue dye, Ann. Nucl. Med. 22(6), 487-494 (2008).

18. H. Niikura, C. Okamura, and J. Akahira, Sentinel lymph node detection in early cervical cancer with combination 99mTc phytate and patent blue, Gynecol. Oncol. 94, 528-532 (2004).

19. A. L. Chernysheva, L. A. Kolomiets, I. G. Sinilkin, V. I. Chernov, and A. Yu. Lyapunov, The choice of surgical treatment in patients with cervical cancer, Malignant Tumor 13(2), 64-70 (2015).

20. Xue-Lian Du, Xiu-Gui Sheng, and Tao Jiang, Sentinel lymph node biopsy as guidance for radical trachelectomy in young patients with early stage cervical cancer, BMC Cancer 11, 157 (2011).

21. A. N. Fader, R. P. Edwards, and M. Cost, Sentinel lymph node biopsy in early-stage cervical cancer: utility of intraoperative versus postoperative assessment, Gynecol. Oncol. 111(1), 13-17 (2008).

22. M. S. Baggish and M. K. Karram, Atlas of Pelvic Anatomy and Gynecologic Surgery (Elsevier Ltd. Press, London, 2009). 